\title{
APROXIMACIÓN HISTÓRICA AL GÉNERO Iguanodon
}

\author{
José Luis SANZ \\ Unidad de Paleontología. Dpto. Biología. Universidad Autónoma. Cantoblan- \\ co 28049 Madrid
}

Sanz, J. L. 2005. Aproximación histórica al género Iguanodon. [Historical approach at genus Iguanodon.] Revista Española de Paleontología, N.E. X, 5-14. ISSN 0213-6937.

\begin{abstract}
The lizard-like model of Iguanodon, as interpreted by Mantell, was refuted by Owen. This mammal-like interpretation was, in turn, refuted by the biped kangaroo-like of Dollo. The present day model of Iguanodon comes from Norman, Galton and Weishampel, and it is considered as an optional biped/quadrupedal dinosaur, depending on the species and ontogenetic development. Iguanodon was a large plant-eater that lived in Eurasia and North America during the Lower Cretaceous (Berriasian- Albian). Its phylogenetic relationships context, within the ornithopod ornithischian dinosaurs, is widely accepted. The family Iguanodontidae (Iguanodon + Ouranosaurus + Altirhinus) is probably paraphyletic, as well as the genus Iguanodon itself. The sociocultural projection of Iguanodon has been determined by the fact of being an "european dinosaur". It was removed of the first movie version of "The Lost World" (1925) and is presented in a "cameo" appearance in "Fantasia". Finally, Disney starred Iguanodon in "Dinosaur" (2000).
\end{abstract}

Key Words: Dinosauria, Ornithopoda, Iguanodon, Osteology, Phylogenetic Relationships, Evolutionary History, Socio-cultural Projection, Lower Cretaceous.

\section{RESUMEN}

El modelo lacertoide de interpretación de Iguanodon por parte de Mantell fue refutado por Owen. A su vez, esta interpretación mamiferoide fue refutada por Dollo a favor de de un modelo bípedo canguroide. La interpretación actual, que procede de Norman, Galton y Weishampel, describe a Iguanodon como un cuadrúpedo/bípedo opcional, dependiendo de las especies y del desarrollo ontogénico. Iguanodon fue un gran fitófago que vivió en Eurasia y América durante el Cretácico Inferior (Berriasiense-Albiense). Su contexto filogenético dentro de los dinosaurios ornitisquios ornitópodos tiene un amplio consenso. La familia Iguanodontidae (que incluye a Iguanodon + Ouranosaurus + Altirhinus) es probablemente parafilética, al igual que el propio género Iguanodon. La proyección sociocultural de Iguanodon ha sido determinada en gran parte por su condición de "dinosaurio europeo". Fue eliminado de la primera versión cinematográfica de "El mundo perdido" (1925) y aparece unos segundos en "Fantasia". Por fin, Disney le convirtió en protagonista de la película "Dinosaurio" (2000).

Palabras clave: Dinosauria, Ornithopoda, Iguanodon, Osteología, Filogenia, Historia evolutiva, Proyección sociocultural, Cretácico Inferior.

\section{INTRODUCCIÓN: MANTELL Y OWEN}

El género Iguanodon (Dinosauria, Ornithopoda) contiene varias especies con numerosas poblaciones que vivieron en Eurasia, América y quizás también África durante el Cretácico Inferior (Berriasiense-Albiense). Iguanodon fue el segundo dinosaurio nominado en la literatura científi ca en la primera mitad del siglo XIX, inmediatamente después de la publicación de Megalosaurus por W. Buckland (1784-1856) en 1824. El material original consiste en siete dientes aislados desgastados procedentes de Til- gate Forest, Sussex, Inglaterra. El médico inglés Gideon Algernon Mantell (1790-1852) describió estos dientes, junto con vértebras y otros restos, en "The Fossils of South Downs" (1822), acreditando el hallazgo a su esposa (Mantell, 1833). La naturaleza de estos materiales era realmente problemática para los conocimientos de la época y Mantell pidió opinión a Georges Cuvier (1769-1832), cuya primera interpretación fue que procedían de un gran mamífero fitófago, probablemente un rinoceronte. Esta interpretación era contradictoria con la identificación cretácica de Mantell de los depósitos de Tilgate Forest. En junio de 1824 Cuvier 
reconsideró su respuesta, proponiendo que los enigmáticos restos procedían de un gran reptil herbívoro. El naturalista S. Stutchbury, en el Hunterian Museum, advirtió a Mantell de la semejanza morfológica de su material con los dientes de la actual iguana terrestre. Esta sugerencia convenció a Mantell de la naturaleza lacertiliana del material, al que denominó Iguanodon (1825), un saurio cuyo tamaño calculó inicialmente en unos 40 pies (12 m). En 1829 F. Holl asignó al género de Mantell una denominación especifica, I. anglicum (corregido posteriormente a I. anglicus), que se convirtió automáticamente en la especie tipo. Tres años más tarde el paleontólogo alemán C.E.H von Meyer (18011869) redenominó al material original de Mantell como $I$. mantelli, nombre ampliamente admitido durante el siglo XIX como especie tipo de Iguanodon. En 1834 se encontró en una cantera de Maidstone (Kent) un esqueleto parcial, desarticulado, que incluía sobre todo huesos apendiculares y evidencia de dentición. Gracias a este hallazgo Mantell pudo proponer la primera reconstrucción conocida de un dinosaurio: un enorme reptil iguanoide arborícola, dotado de un cuerno nasal (Fig. 1.a). Esta interpretación de Mantell, seguidor de la Teología Natural, fue ampliamente refutada por Richard Owen (1804-1892) dentro del marco conceptual y metodológico de la Filosofía Natural. La evidencia morfológica e incluso histológica de Iguanodon, y otros grandes reptiles del Weald, llevó a Owen a la propuesta del taxón Dinosauria (1842), concebidos como pesados cuadrúpedos de aspecto mamiferoide (Fig. 1.b).

\section{LOS HALLAZGOS DE BERNISSART: DOLLO}

En 1878 se iniciaron en una mina de carbón de Bernissart (Bélgica) una serie de descubrimientos consistentes en enormes carcasas que fueron identificadas como Iguanodon por Van Beneden y nominadas I. benissartensis por G. A. Boulanger en 1881. No obstante, fue el paleontólogo franco-belga Louis Dollo (1857-1931) quien trabajó de manera más activa sobre el extraordinario material de Bernissart, finalmente compuesto por 24 esqueletos articulados más o menos completos, así como diversos individuos de preservación parcial. Dollo supervisó la difícil preparación de los esqueletos, así como su montaje, el primero de un dinosaurio que se realizaba en Europa. Dollo propuso el término "Paleontología Etológica" (Paleobiogía) para conceptualizar y sistematizar la parte de la Paleontología que se ocupa del estudio de la forma de vida de los organismos del pasado remoto. Sus herramientas de análisis, que el paleontólogo franco-belga utilizó con una sorprendente dimensión actual, eran básicamente la anatomía comparada y la morfología funcional. De esta forma, Dollo refutó el modelo de Owen para Iguanodon y propuso, mediante una detallada comparación con las aves actuales, que este género tenía una disposición típicamente bípeda (Dollo; 1883, 1884) (Fig. 1.c-1.e). No obstante, de acuerdo con Owen, Dollo sugirió que Iguanodon sería un animal con hábitos esencialmente anfibios (Fig. 1.f).

\section{LAS ESPECIES DE Iguanodon}

Entre todos los individuos hallados en Bernissart destaca un pequeño adulto que fue identificado como I. mantelli. Norman (1986) refirió este individuo a I. atherfieldensis, propuesto por Hooley (1925) para nombrar una relativamente pequeña especie de este género (unos $5 \mathrm{~m}$ ). El material original procede de las "Wealden Shales" ,cerca de Atherfield Point, isla de Wight. Las dos especies de Iguanodon de Bernissart fueron interpretados como dimorfos sexuales por Nopcsa (1929). Aunque rebatida por Norman, esta hipótesis todavía tiene seguidores (Chapman et al., 1997). En 1952 el paleontólogo ruso A.K. Rozhdestvensky propuso la especie I. orientalis basada en un maxilar con dientes, escápula y costillas procedentes de Khmarin-Khural, desierto de Gobi, Mongolia. I. orientalis ha sido considerado como un nomen dubium por Norman $(1986,1996)$. Galton \& Jensen (1979) describieron con un fragmento maxilar de Utah (EEUU) la nueva especie I. ottingeri, cuyo estatus ha sido cuestionado por Norman (1986) y Weishampel \& Bjork (1989). Estos dos últimos autores son los responsables del taxón I. lakotaensis. El material procede de la formación Lakota (Barremiense?), en Dakota del Sur (EEUU) y consiste en un cráneo prácticamente completo y dos vértebras. Según Weishampel \& Bjork (1989) I. lakotaensis comparte con el clado Igua-

Figura 1. Reconstrucción histórica de Iguanodon. a, Modelo lacertoide de Mantell (en "Primitive World", de F. Unger, 1851, fide Rudwick,1992). b, Modelo mamiferoide de Owen (1853). c, Modelo canguroide inspirado en los trabajos de Dollo (Lankester, 1905). d, Bípedo corredor de Heilmann, (1926). e, Hábitos semejantes a los de una jirafa (Heilmann, 1926). f, Hábitos acuáticos según la propuesta de Dollo (Wilfharth, 1949). g, Iguanodon canguroide de extremidades posteriores verticales. Este modelo fue dominante durante los años 1950-60 (Augusta \& Burian, 1961).

Historical reconstruction of Iguanodon. a, Lizard-like model of Mantell (in "Primitive World", by F. Unger, 1851, fide Rudwick, 1992). b, Mammalian-like model of Owen (1853). c, Kangaroo-like model inspired by the works of Dollo (Lankester, 1905). d, Running biped of Heilmann, (1926). e, Similar habits to those of a giraffe (Heilmann, 1926). $f$, Aquatic habits according to the proposal of Dollo (Wilfharth, 1949). g, Kangaroo-like Iguanodon with vertical hind limbs. This model was dominant during the years 1950-60 (Augusta \& Burian, 1961). 


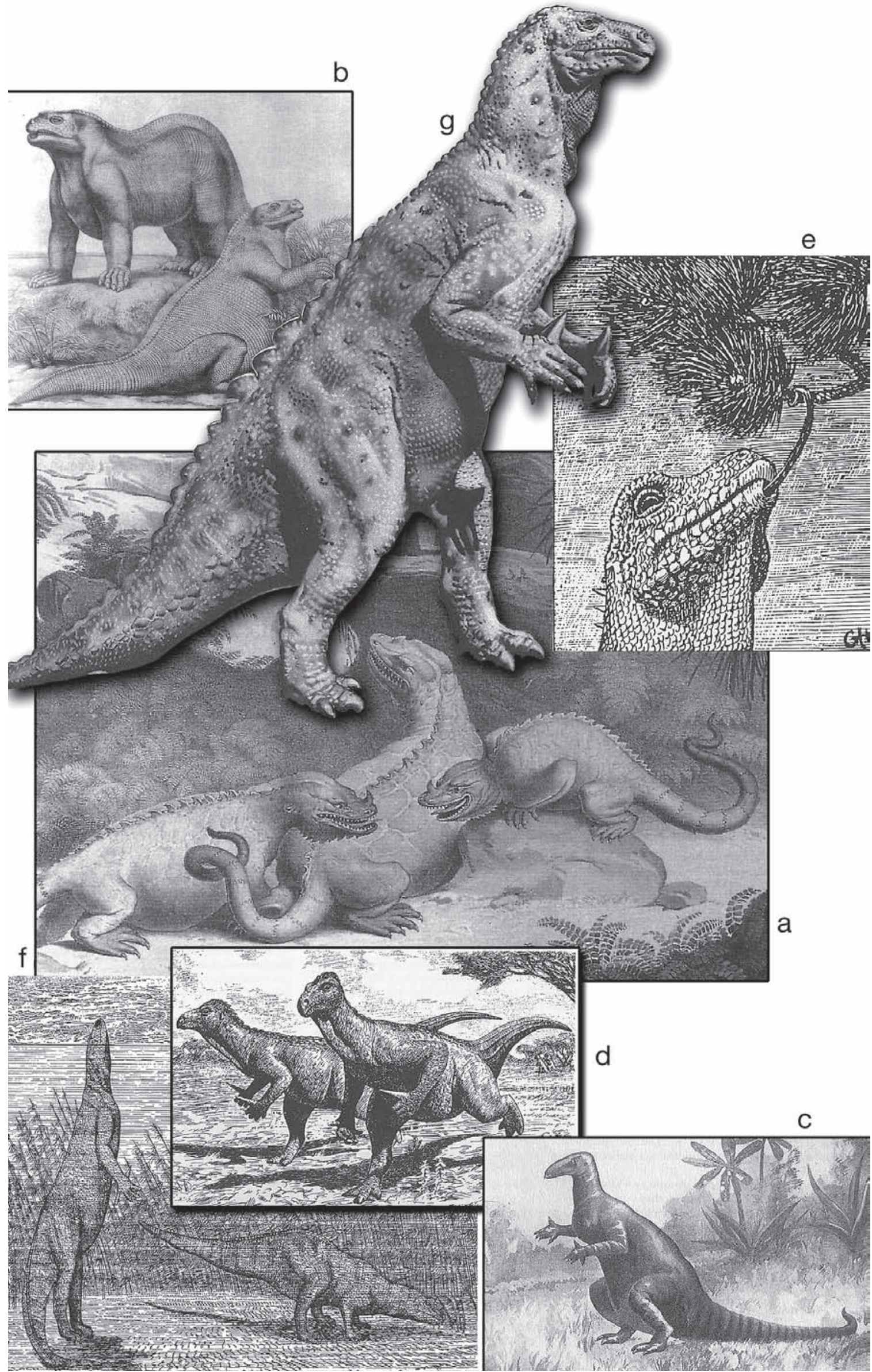


nodon (esencialmente I. atherfieldensis $+I$. benissartensis) las siguientes sinapomorfias: esqueleto facial largo y profundo, pico premaxilar moderadamente expandido, fuerte denticulación del premaxilar y predentario, en el orbitoesfenoides, separación de los forámenes de salida del nervio craneal IV de las de los III y V y margen dorsal del proceso paroccipital redondeado, sin plataformas en el contacto dorsal con el escamoso. No obstante, I lakotaensis presenta claras diferencias con I. atherfieldensis e I. benissartensis, como la existencia de un foramen anteorbitario de gran tamaño, dientes maxilares más pequeños, con menor numero de posiciones, o la ausencia de un diastema entre la dentición del dentario y el predentario (considerado como una sinapomorfía en reversión). En 1986 Norman designó el diente dentario del séptimo sintipo figurado por Mantell (pieza BMNH 2392 del catálogo del Natural History Museum, Londres) como lectotipo de la especie del género (I. anglicus). Sin embargo, Charig \& Chapman (1998) indicaron que esta pieza no podía ser identificada con seguridad en las figuras publicadas por Mantell (1825), solicitando a la Comisión Internacional de Nomenclatura Zoológica la designación de I. benissartensis Boulenger in Beneden 1881 como especie tipo. Esta petición tuvo una respuesta favorable en marzo de 2000 (opinión 1947).

\section{OSTEOLOGÍA Y MODO DE VIDA}

Iguanodon es uno de los géneros de dinosaurios con morfología esquelética mejor conocida (Dollo, 1882, 1883, 1884; Hooley, 1925; Norman, 1980, 1986; Sanz et al., 1982, 1984). A partir de la década de 1980 las nuevas conclusiones aportadas por la sistemática filogenética, el análisis morfofuncional y el registro icnológico cambiaron radicalmente nuestra percepción sobre los hábitos de vida de este dinosaurio. La arquitectura general del cráneo tiene un aspecto equino, con un largo desarrollo facial que acaba en una región rostral especializada. El premaxilar carece de dientes y se abre en paleta. La sínfisis dentaria está coronada por un predentario denticulado. Todo parece indicar la presencia en vida del animal de una ranfoteca que probablemente sería funcionalmente importante durante el ramoneo. Por detrás del pico aparece un diastema. Las series inferior y superior están constituidas por una compleja batería dental. El número de posiciones dentales es mayor que en la condición primitiva representada, por ejemplo, por Camptosaurus (13 maxilares/14 dentarios, frente a 29/25 en I. benissartensis). Las mandíbulas son funcionalmente isognatas y la oclusión es céntrica, semejante a la de los mamíferos. El plano oclusal se sitúa muy por debajo de la articulación cráneo-mandibular (cuadrado-articular). El reemplazamiento dental es "en echelon" y la batería mantiene siempre una desarrollada superficie oclusal de masticación. Esta disposición explica la existencia, en general, de una única faceta de desgaste en cada diente. La morfología dental es característica, con coronas (especialmente en la región yugal) altas y lanceoladas, frecuentemente asimétricas, con una sola capa de esmalte (situada bucalmente en los dientes maxilares y lingualmente en los dentarios). El esmalte forma crestas denticuladas mesiales y distales. Las coronas maxilares están ornamentadas con una fuerte cresta primaria de posición ligeramente distal y secundarias adyacentes. Las coronas dentarias tienen dos crestas principales limitadas mesial y distalmente por otras secundarias. La aparición de baterías dentales y las complejas relaciones artrológicas del cráneo indican una mecánica trófica derivada en Iguanodon. A partir de la condición primitiva, que aparece en ornitópodos basales, los euornitópodos se caracterizan por un cráneo en el que se reestructuran las relaciones entre maxilar-premaxilar, yugal-postorbitario, cuadrado-cuadradoyugal y basipterigoides-pterigoides. El complejo maxilo-palatino es desplazado lateralmente por el movimiento vertical oclusivo de los dentarios (pleurocinesis) (Weishampel, 1984; Weishampel \& Norman, 1989). La evidencia de los patrones de microestrías de desgaste en las facetas de dientes de Iguanodon es congruente con el modelo pleurocinético. Este proceso genera un recurso mecánico de trituración del alimento análogo al desplazamiento lateral mandíbulo-craneal propio de los mamíferos. La elevación vertical de los dentarios se producía mediante la contracción de los músculos aductores de la mandíbula. El gran desarrollo relativo de la apófisis coronoides en Iguanodon indica una gran capacidad de cierre cráneo-mandibular (Wu \& Russell, 1997). El sofisticado proceso masticador en Iguanodon (y otros ornitópodos) estaba asistido por dos factores cuya importancia puede ser subrayada por dos homoplasias compartidas con mamíferos fitófagos actuales: un diastema por delante de los dientes yugales y carrillos que cierran la boca. La existencia de mejillas en dinosaurios ha sido, no obstante, puesta en duda recientemente (Papp \& Witmer, 1998). La eventual definitiva refutación de la hipótesis de existencia de mejillas en dinosaurios no invalidaría, en cualquier caso, la hipótesis pleurocinética. Determinados caracteres craneales en Iguanodon, como la reducción de la ventana preorbitaria, pueden estar en relación con la historia de la especialización trófica en las formas más derivadas de ornitópodos. El sistema de masticación pleurocinética necesitaría de una región maxilo-lacrimal estructuralmente reforzada, con reducción del seno anteorbital (Witmer, 1997). Otras peculiaridades craneales de Iguanodon, como el gran desarrollo de las narinas externas, tiene una interpretación paleobiológica más problemática, y puede correlacionarse con patrones de display, regulación térmica y/o mecánica respiratoria. Por último, tampoco es evidente que Iguanodon y otros ornitópodos, tuvieran una membrana timpánica, que pudo estar situada entre el cuadrado y la región distal del proceso paroccipital (Norman, 1986). 
El esqueleto axial de Iguanodon comienza con un proatlas cuya función puede ser ayudar a mantener el cráneo en una posición horizontal, previniendo posibles dislocaciones del cuello en el cóndilo occipital (Norman, 1986). Las vértebras cervicales tienen neurapófisis relativamente bajas y centros fuertemente opistocélicos, caracteres que indican un cuello con una gran movilidad potencial. La serie dorsal típica tiene centros anfiplanos y altas neurapófisis. El sacro contiene de seis a siete elementos, mientras la serie caudal puede tener más de 45 vértebras. Gran parte de las neurapófisis (aproximadamente desde la décima cervical a la vigésima caudal) están conectadas por tendones osificados. En I. benissartensis, además, estos elementos aparecen también en procesos transversos de dorsales y sacras (Norman, 1980). La aparición de tendones osificados fue un carácter de primera magnitud para redefinir la posición sub-horizontal del esqueleto axial en Iguanodon y otros ornitópodos derivados (Galton, 1970). De hecho, la forzada posición caudal de la reconstrucción de Iguanodon por parte de Dollo estaba sesgada por las ideas de Leidy. La redefinición postural de Iguanodon implica la posibilidad de que fuera capaz de realizar un marcha cuadrúpeda. Esta hipótesis ha sido refrendada por el registro icnológico (Moratalla et al., 1992, 1994) que además indica un bajo coste energético para el paso de la estación bípeda a la cuadrúpeda. En consonancia con esta versatilidad locomotora Iguanodon tiene un cuarto trocánter femoral de gran desarrollo (musculatura caudofemoral) y una singular arquitectura del autópodo anterior. El carpo está osificado en huesos paralepipédicos, lo que reduciría la movilidad de la mano pero reforzaría la capacidad de carga de la muñeca. El primer dedo de la mano diverge ortogonalmente del eje de desarrollo de la extremidad, y está rematado por una falange ungueal en forma de espolón, probablemente cubierta en vida por un agudo estuche córneo. Los dígitos II a IV tienen una morfología "normal" (primitiva), con falanges ungueales en forma de pezuña en los dos más internos. Aunque su capacidad de flexión sería limitada, la extensión sería probablemente similar a la de los dedos del pie. El registro icnológico indica que los dedos III y IV estaban unidos en una única estructura solidaria (Moratalla et al., 1992). Por último, el dedo V, oponible, puede flexionarse contra la palma de la mano, que adquiere así funciones prensiles. En definitiva, la mano de Iguanodon debió ser una estructura de gran versatilidad involucrada en funciones de locomoción, prensión y quizás también exhibición y defensa.

Recordemos brevemente que los modelos generales de interpretación de Iguanodon han sido sucesivamente: lacertoide (Mantell), mamiferoide (Owen) y bípedo canguroide anfibio (Dollo). De todo lo arriba expuesto puede deducirse que el modelo actual es el de un bípedo/cuadrúpedo opcional (dependiendo de factores ontogenéticos y también de diferencias específicas) terrestre (Fig. 2). La presencia de Iguanodon en las cadenas tróficas de los ecosistemas continentales del Cretácico inferior es muy poco evidente, aunque se ha señalado el hallazgo de material juvenil de este ornitópodo dentro de la caja torácica del terópodo Baryonyx (Charig \& Milner, 1997). Quizás una manera de hacer frente a la presión de los depredadores fuera la aparición de hábitos gregarios, que han sido verificados mediante el registro icnológico (Lockley \& Matsukawa, 1999). Los fenómenos de osteoartritis en Iguanodon debieron ser relativamente frecuentes. Rothschild (1997) describe recrecimientos de la superficie articular de los huesos del tobillo en dos casos sobre 39 escrutados. Paradójicamente esta patología indica un tobillo dotado de gran movilidad. El yacimiento número cinco de Mas de la Parreta en Morella ha proporcionado recientemente un interesante caso de una vértebra afectada por una fusión costal al proceso transverso (Cambra Moo et al., 2002) (Fig. 3). El género Iguanodon ha ingresado recientemente en el dominio de la Paleontología molecular, con el hallazgo de material proteínico en una costilla (Embery et al., 2003).

\section{FILOGENIA}

El contexto filogenético general del género Iguanodon, dentro de los dinosaurios ornitisquios ornitópodos, tiene un amplio consenso. Es patente la presencia de sinapomorfías ornitisquias, como el predentario, palpebral o la estructura de la pelvis. Iguanodon comparte novedades evolutivas con otros ornitópodos, como el proceso paroccipital en forma de media luna o el desarrollo del proceso lateral del premaxilar para contactar con el lacrimal. La topología general del clado Ornithopoda también tiene un cierto consenso (Fastovsky \& Weishampel, 1996; Norman, 1998, 2002; Sereno, 1999). Los heterodontosaurios constituyen el grupo más basal de Ornithopoda, denominándose Euornithopoda al antecesor común del resto de los linajes conocidos y todos sus descendientes. Los euornitópodos se caracterizan por la aparición de los cráneos pleurocinéticos y determinadas modificaciones pélvicas y femorales. Su grupo más basal son los hipsilofodóntidos. La posición de los driosáuridos y el género Tenontosaurus es debatida, pero se acepta el clado Ankylopollexia (Camptosauria sensu Norman, 1998) definido como el antecesor común de Camptosaurus y los hadrosaurios y todos sus descendientes. Una de las novedades evolutivas más evidentes de este clado es la modificación de la arquitectura del carpo, asi como de la morfología dental y falangeal de la mano. La composición taxonómica del clado Iguanodontoidea (sensu Norman, 1998, incorrectamente denominado "Iguanodontia", fig. 35, pag. 334) es equivalente a Hadrosauriformes (sensu Sereno, 1999), pero no así la topología de cada clado. Para Sereno Probactrosaurus es el taxón más basal, mientras que Norman lo considera el 


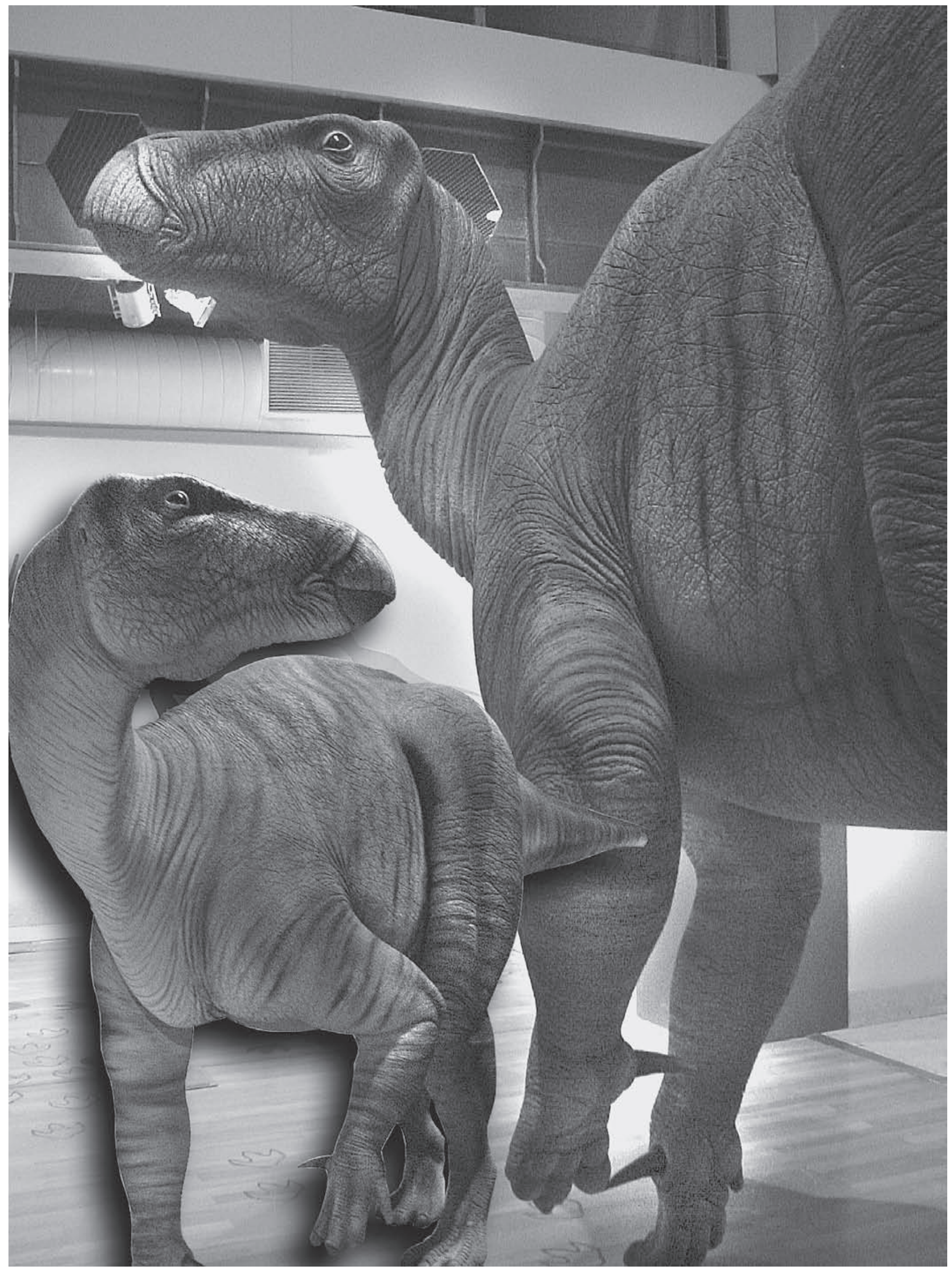


grupo hermano de los hadrosaurios. El clado Iguanodontoidea está diagnosticado por diversas novedades evolutivas, como un diastema bien desarrollado, una compleja articulación yugo-maxilar, proceso coronoides muy alto y falanges ungueales de los dedos dos y tres de la mano en forma de pezuña, acortamiento del proceso postpúbico o la aparición de un cuarto trocánter femoral de morfología subtriangular, no pendant. Norman (1998) defiende la monofilia de la familia Iguanodontidae, definida por el antecesor común de Altirhinus, Ouranosaurus e Iguanodon y todos sus descendientes. Sin embargo, Sereno (1986) y Head (2001) proponen el carácter no natural de este taxón. Finalmente Norman (2002) admite el estatus parafilético de los iguanodóntidos, formados por una estructura stem que sucesivamente (Iguanodon-Ouranosaurus-Altirhinus) se acerca a la condición de las formas pre-hadrosaurianas (Protohadros, Probactrosaurus). El género Lurdusaurus (Cretácico Inferior, Níger), considerado por Taquet \& Russell (1999) como un iguanodóntido, se sitúa en la base de Ankylopollexia, fuera de Iguanodontoidea (Norman, 2002). A pesar de presentar caracteres muy derivados (por ejemplo la ausencia de ventana anteorbital), Wang \& Wu (2001) incluyen a Jinzhousaurus (Cretácico Inferior, China) dentro de los iguanodóntidos. Se trata, probablemente, de una forma cercanamente situada a la base de los Hadrosauria. El carácter monofilético de Iguanodon ha sido propuesto reiteradamente (Norman, 1986; Weishampel \& Bjork, 1989). No obstante, los resultados del reciente análisis de Norman (2002) indican la parafilia del género (deducible por la figura 34 , pag. 139 , pero no comentada en el texto). La matriz taxones x caracteres (apéndice 1, pag. 143) no incluye ningún rasgo autapomórfico en Iguanodon (a pesar de la diagnosis genérica propuesta por el mismo autor en 1986). Upchurch et al. (2002) también incluyen una hipótesis general de relaciones de parentesco en ornitisquia en la que aparece Iguanodon como parafilético (Ouranosaurus de posición intermedia entre I. atherfieldensis e I. benissartensis).

\section{PROYECCIÓN SOCIOCULTURAL}

Independientemente de su estatus sistemático Iguanodon es uno de los dinosaurios de mayor proyección socio cultural (nacida a mediados del siglo XIX), aunque fuertemente condicionada por su carácter de "dinosaurio europeo". En 1851 se inauguró en Londres la gran exposición universal. Al año siguiente se ofreció a Mantell dirigir la reconstrucción de dinosaurios para la reinaugu-

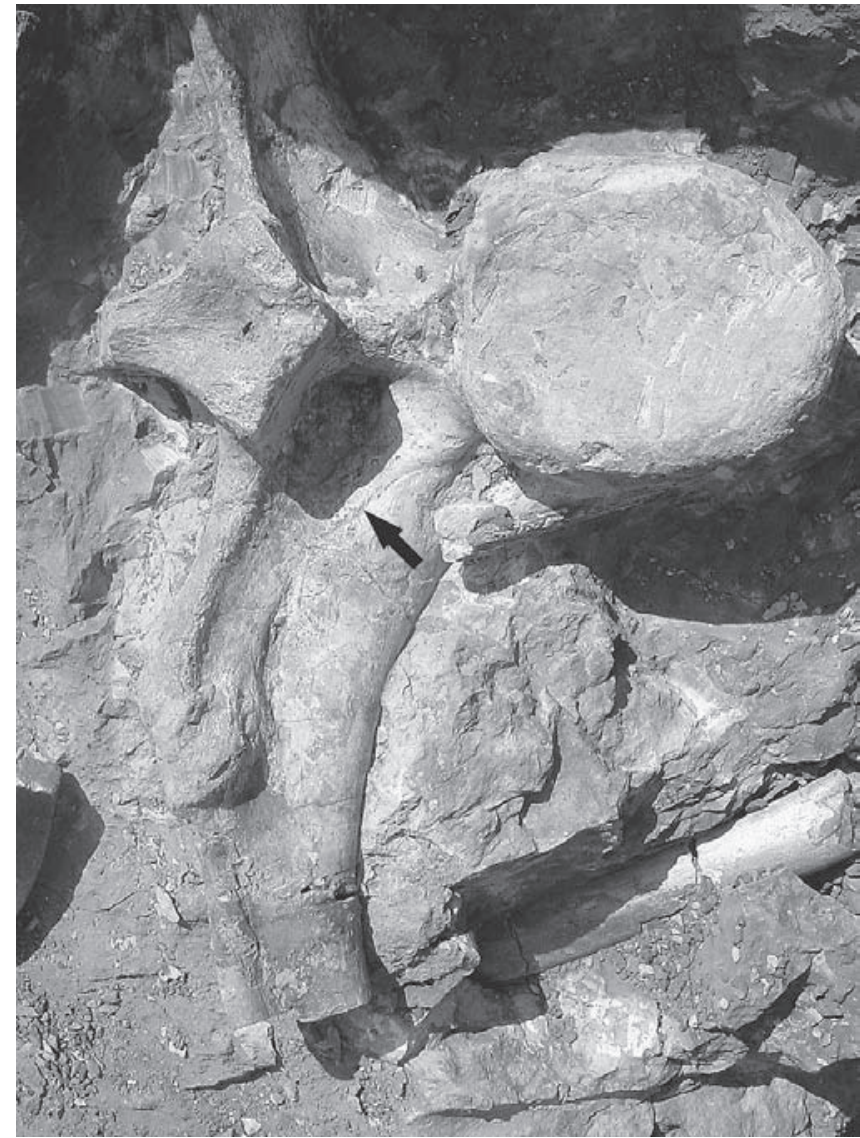

Figura 3. Vértebra de Iguanodon in situ (vista posterior). Yacimiento $n^{\circ} 5$ de Mas de la Parreta (Morella, Castellón). La flecha indica un surco situado en la zona de fusión de la costilla izquierda con la apófisis transversa. Vertebra of Iguanodon placed in situ (posterior view). Mas de la Parreta (Morella, Castellón) locality $n^{\circ} 5$. The arrow indicates a furrow located at the fusion zone of the left rib with the transversa apophysis.

ración del "Crystal Palace" en Sydenham en 1854. El consorcio estaba dispuesto a realizar una gran inversión en el proyecto (3000 a 4000 libras). A pesar de que era la gran oportunidad de Mantell para reivindicar públicamente su querido Iguanodon, declinó la oferta, según Cadbury (2000) por serios problemas de salud (estaba casi inválido por un accidente de 1841). La interpretación de Dean (1999) es diferente: “...he disliked the idea of life-sized models-as opposed to more scientific displays". En definitiva, la popular imagen victoriana de Iguanodon como un pesado mamiferoide, fruto de la colaboración OwenWaterhouse Hawkins, pudo haber sido muy diferente (no

Figura 2. Maqueta a escala 1:1 de Iguanodon. Realizada por Javier Hernández para el Museo “Temps de Dinosaures" de Morella (Castellón).

1:1 scale model of Iguanodon. Made by Javier Hernandez to the Museum "Temps de Dinosaures" in Morella (Castellón). 
sólo en morfología, también en tamaño). Científico y artista se asociaron para generar las enormes esculturas, que eran primero dibujadas, luego esbozadas mediante una maqueta y finalmente construidas con ladrillos, hierro, cerámica y hormigón. De las cuatro estatuas de dinosaurios dos son de Iguanodon en diferentes actitudes, erguido y agachado. Las razones de esta discriminación positiva hacia el dinosaurio de Mantell no parecen claras. Es posible que Owen quisiera ilustrar su idea sobre su reciente taxón Dinosauria en el género mejor conocido de los tres. Owen y W. Hawkins fueron los anfitriones de la famosa cena del dinosaurio en la Nochevieja de 1853 que tuvo una amplia difusión en la prensa de la época. Iguanodon fue un dinosaurio "típicamente inglés" (la villa de Maidstone llegó a incluirlo en su escudo heráldico en 1946) hasta las dos últimas décadas del siglo XIX. Los trabajos de Dollo sobre el material de Bernissart popularizaron un Iguanodon "belga" erguido, de aspecto canguroide, que puede verse en la película checa "Viaje a la prehistoria" ( "Cesta do Praveku”, K. Zeman, 1954) e incluso rastrearse hasta el cómic más popular norteamericano de mediados de los años 1970. La interpretación de Dollo, el dinosaurio canguro-jirafa que vivía en rebaños, es la que se instala en "El mundo perdido" ("Lost World", A. C. Doyle, 1912). Una de las circunstancias que indujeron al autor de Sherlock Holmes a escribir el relato sobre la pervivencia de dinosaurios en Sudamérica fue el hallazgo de icnitas de iguanodóntidos en Sussex, cerca de su casa que llegó a colocar en su sala de billar (Sanz, 2002). Los paleontólogos de ficción descubren huellas frescas de Iguanodon y más tarde los animales vivos que son utilizados como ganado por los hombres primitivos de la meseta perdida. Parece bastante significativo que Iguanodon fuera eliminado de la primera versión cinematográfica de "El mundo perdido" ("Lost World", H. Hoyt, 1925) en la que sólo aparecen dinosaurios norteamericanos. Los científicos que exploran el mundo subterráneo prehistórico de "Plutonia" (Obruchev, 1924) se alimentan de carne de Iguanodon, enormes reptiles "semejantes a canguros" de color verde oscuro con manchas parduscas. El género Iguanodon es uno de los dinosaurios pertenecientes al dominio criptozoológico. Los dragones representados en la puerta de Istar, en Babilonia, no eran animales mitológicos, sino representaciones de dinosaurios iguanodóntidos, parecidos a los hallados en Bélgica, todavía vivos en el siglo $\mathrm{V}$ antes de nuestra era en Mesopotamia. Esta arriesgada propuesta fue realizada en 1913 por el arqueólogo alemán Robert Koldeway (Heuvelmans, 1978).

A finales de los años 1980 una iniciativa privada, "Recherche des Iguanodons", creada para reabrir las minas en Bernissart, tuvo un cierto eco mediático. Diez años después la Comisión Europea financió la creación de un Iguanodon robótico con fines esencialmente didácticos y comerciales, un dinosaurio europeo animatrónico (Papantoniu et al.,
1999). La eliminación de Iguanodon de la primera gran película norteamericana de dinosaurios parece haber generado su ausencia en todas las grandes producciones de Hollywood del género (excepto "Fantasia", S. Armstrong, B. Roberts, P. Satterfield, H. Luske, J. Handley, F. Beebe, N. Ferguson \& W. Jackson, 1940) en la que tres iguanodóntidos canguros saltan durante unos tres segundos delante de un tiranosaurio. Esta discriminación hacia Iguanodon por parte de Hollywood se corrige en el año 2000 con la película "Dinosaurio" ("Dinosaur", Eric Leighton) cuyo protagonista es un joven Iguanodon cuadrúpedo (de nombre Aladar) dotado de un gran corazón (carácter no preservable en el registro fósil y por tanto imposible de evaluar, al menos de momento, por los paleontólogos).

\section{REFERENCIAS}

Augusta, J. \& Burian, Z. 1961. Los animales prehistóricos. Queromón editores. Madrid. México.

Cadbury, D. 2000. Dinosaur Hunters: A True Story of Scientific Rivalry and the Discovery of the Prehistoric World. Forth Estate, London.

Cambra Moo, O., Ortega, F., Gasulla, J. M. \& Yagüe, P. 2002. "Diga 33". Aplicación del diagnóstico diferencial a una patología vertebral en Iguanodon (Cretácico inferior, Morella, Castellón).Congreso Internacional sobre Dinosaurios y otros Reptiles mesozoicos de España. Resúmenes, 9.

Chapman, R.E., Weishampel, D., Hunt, E. \& Rasskin-Gutman, D. 1997. Sexual dimorphism in dinosaurs. Dinofest International Proceedings, 83-93.

Charig,A.J. \& Milner,A.C. 1997. Baryonyx walkeri, a fisheating dinosaur from the Wealden of Surrey. Bulletin of the Natural History Museum London (Geology), 53, 11-70.

Charig, A. \& Chapman, S. 1998. Case 3037. Iguanodon bernissartensis Boulenger in Beneden, 1881: proposed as the type species of Iguanodon Mantell, 1825, and proposed designation of a lectotype. Bulletin of Zoological Nomenclature, 55, 99-104.

Dean, D.R. 1999. Gideon Mantell and the Discovery of dinosaurs. Cambridge University Press, Cambridge.

Dollo, L. 1882. Premiere note sur les dinosauriens de Bernissart. Bulletin du Musee Royal d'Histoire Naturelle de Belgique, 1, 161-180.

Dollo, L. 1883. Troisieme note sur les dinosauriens de Bernissart. Bulletin du Musee Royal d'Histoire Naturelle de Belgique, 2, 85-126.

Dollo, L. 1884. Cinquieme note sur les dinosauriens de Bernissart. Bulletin du Musee Royal d'Histoire Naturelle de Belgique, 3, 120-140.

Embery, G., Milner, A. C., Waddington, R. J., Hall, R. C., Langley, M. S. \& Milan, A. M. 2003. Identification of Proteinaceous Material in the Bone of the Dinosaur Iguanodon. Connective Tissue Research, 44, 41-46.

Fastovsky, D.E. \& Weishampel, D.B. 1996. The Evolution 
and Extinction of the Dinosaurs. Cambridge University Press, Cambridge.

Galton, P. M. 1970. The posture of hadrosaurian dinosaurs. Journal of Paleontology, 44, 464-473.

Galton, P.M. \& Jensen, J.A. 1979. Remains of ornithopod dinosaurs from the Lower Cretaceous of North America. Brigham Young University Geological Studies, 25, 1-10.

Head, J. J. 2001. A reanalysis of the phylogenetic position of Eolambia caraljonesa (Dinosauria, Iguanodontia). Journal of Vertebrate Paleontology, 21, 392-396.

Heilmann, G. 1926. The Origin of Birds. Witherby, London.

Heuvelmans, B. 1978. Les Derniers Dragons d'Afrique. Plon, Paris.

Hooley, R. W. 1925. On the skeleton of Iguanodon atherfieldensis sp. nov. Quarterly Journal of the Geological Society, 81, 1-60.

Lankester, E. R. 1905. Extinct Animals. Archibald Constable, London.

Lockley, M. G. \& M. Matsukawa. 1999. Some observations on trackway evidence for gregarious behavior among small bipedal dinosaurs. Palaeogeography, Palaeoclimatology, Palaeoecology, 150, 25-31.

Mantell, G. 1822. The fossils of the South Downs; or, Illustrations of the Geology of Sussex. Lupton Relfe, London.

Mantell, G. A. 1825. Notice on the Iguanodon, a newly discovered fossil reptile, from the sandstone of Tilgate Forest, in Sussex. Philosophical Transactions of the Royal Society, London, 115, 179-186.

Mantell, G. 1833. The Geology of the South-East of England. Longman, London

Moratalla, J.J. Sanz, J.L, Jimenez, S. \& Lockley, M.G. 1992. A quadrupedal ornithopod trackway from the Lower Cretaceous of La Rioja (Spain): Inferences on gait and hand structure. Journal of Vertebrate Paleontology, 12, 150-157.

Moratalla, J.J. Sanz, J.L \& Jimenez, S. 1994. Dinosaur tracks from the Lower Cretaceous of Regumiel de la Sierra (province of Burgos, Spain): Inferences on a new quadrupedal ornithopod trackway. Ichnos, 3, 89-97

Nopcsa, F. 1929. Sexual differences in ornithopodous dinosaurs. Palaeobiologica, 2, 187-201.

Norman, D.B. 1980. On the Ornithischian Dinosaur Iguanodon bernissartensis from the Lower Cretaceous of Bernissart (Belgium). Memoire No. 178 de L'Institut Royal des Scienes Naturelles de Belgique, Bruxelles.

Norman, D.B. 1986. On the anatomy of Iguanodon atherfieldensis (Ornithischia: Ornithopoda). Bulletin du Musee Royal d'Histoire Naturelle de Belgique, 56, 281-372.

Norman, D. B. 1996. On Mongolian ornithopods (Dinosauria: Ornithischia). 1. Iguanodon orientalis Rozhdestvensky 1952. Zoological Journal of the Linnaean Society, 116, 303-315.

Norman, D.B. 1998. On Asian ornithopods (Dinosauria: Ornithischia). 3. A new species of iguanodontid dinosaur. Zoological Journal of the Linnaean Society, 122, 291-348.

Norman, D.B. 2002. On Asian ornithopods (Dinosauria: Orni- thischia). 4. Probactrosaurus Rozhdestvensky, 1966. Zoological Journal of the Linnaean Society, 136, 113-144.

Obruchev, V. A. 1924. Plutonia. Ediciones en lenguas extranjeras, Moscú.

Opinion 1947. 2000. Iguanodon Mantell (Reptilia, Ornithischia): Iguanodon bernissartensis Boulenger in Beneden, 1881: designated as the type species, and a lectotype designated. Bulletin of Zoological Nomenclature, 57, 61-62.

Owen, R. 1842. Report on British fossil reptiles, Part II. Reports of the British Association for the Advance of Science, 1841, 60-294.

Papantoniou, V., Avlakiotis, P. \& Alexander, R.M. 1999. Control of a robot dinosaur. Philosophical Transactions of the Royal Society of London, 354, 863-868.

Papp, M. J., \& Witmer, L. M. 1998. Cheeks, beaks, or freaks: a critical appraisal of buccal soft-tissue anatomy in ornithischian dinosaurs. Journal of Vertebrate Paleontology, 18 (3:Suppl.), 69A

Rozhdestvensky. A. K. 1952. [Descubrimiento de un iguanodóntido en Mongolia]. Doklady Akademy Nauk S.S.S.R. , 84,1243-1246 (en ruso).

Rudwick, M. J. S. 1992. Scenes from Deep Time. University of Chicago Press, Chicago.

Sanz J. L. 2002. Starring T. Rex!: Dinosaur Mythology and Popular Culture. Indiana University Press.

Sanz, J.L., Casanovas-Cladellas, M.L. \& Santafé-Llopis, J.V. 1982. Paleontología. In: Geología y Paleontología (Dinosaurios) de las Capas rojas de Morella (Castellón, España) (eds. J. V. Santafé-Llopis, M. L. Casanovas-Cladellas, J. L. Sanz \& S. Calzada). Diputación provincial de Castellón y Diputación de Barcelona, 69-169.

Sanz, J. L., Casanovas, M. L. \& Santafé, J. V. 1984. Iguanodóntidos (Reptilia, Ornithopoda) del yacimiento del Cretácico inferior de San Cristobal (Galve, Teruel). Acta Geológica Hispánica, 19,171-176.

Sereno, P.C. 1986. Phylogeny of the Bird-Hipped Dinosaurs (Order Ornithischia). National Geographic Research, 2 (2), 234-256.

Sereno, P. C. 1999. The evolution of dinosaurs. Science, 284, 2137-2147.

Taquet, P. \& Russell, D. A. 1999. A massively-constructed iguanodont from Gadoufaoua, Lower Cretaceous of Niger. Annales de Paleontologie, 85, 85-96.

Upchurch, P., Hunn, C. A. \& Norman, D. B. 2002. An analysis of dinosaurian biogeography: evidence for the existence of vicariance and dispersal patterns caused by geological events. Proceedings of the Royal Society of London, Series B, 269, 613-621.

Wang, X. \& Xu, X. 2001. A new iguanodontid (Jinzhousaurus yangi gen. et sp. nov.) from the Yixian Formation of western Liaoning, China. Chinese Science Bulletin, 46 (19), 1669-1672.

Weishampel, D.B. 1984. Evolution of jaw mechanisms in ornithopod dinosaurs. Springer-Verlag, Berlin, Heidelberg, New York, Tokyo.

Weishampel, D. B. \& Bjork, P. R. 1989. The first indisputable remains of Iguanodon (Ornithischia: Ornithopoda) from 
North America: Iguanodon lakotaensis n. sp. Journal of Vertebrate Paleontology, 9, 56-66.

Wilfarth, M. 1949. Die lebenweise der Dinosaurier. Stuttgart.

Witmer, L.M. 1997. Craniofacial Air Sinus Systems. In: En- cyclopedia of Dinosaurs (eds. J. Currie \& K. Padian). Academic Press, San Diego, California/London, 151-159

Wu X.-C. \& Russell, A.P. 1997. Functional Morphology. In: Encyclopedia of Dinosaurs (eds. J. Currie \& K. Padian). Academic Press, San Diego, California/London, 258-268. 